\title{
Traduire
}

Une autre perspective sur r tr traduction

Revue française de la traduction

229 | 2013

L'environnement, une spécialisation durable?

\section{L'art de citer, ou les dérives de la négligence}

chevaux de poste et texte à demi publié

Jean Delisle

\section{(2) OpenEdition}

Journals

Édition électronique

URL : http://journals.openedition.org/traduire/605

DOI : $10.4000 /$ traduire.605

ISSN : 2272-9992

Éditeur

Société française des traducteurs

Édition imprimée

Date de publication : 15 décembre 2013

Pagination : 100-102

ISSN : 0395-773X

Référence électronique

Jean Delisle, "L'art de citer, ou les dérives de la négligence », Traduire [En ligne], 229 | 2013, mis en ligne le 28 mai 2014, consulté le 25 septembre 2020. URL : http://journals.openedition.org/traduire/ 605 ; DOI : https://doi.org/10.4000/traduire.605

Ce document a été généré automatiquement le 25 septembre 2020. 


\section{L'art de citer, ou les dérives de la négligence}

Chevaux de poste et texte à demi publié

Jean Delisle

1 Une citation orpheline de sa source est toujours suspecte et oblige le traducteur à redoubler de vigilance. L'académicien Émile Faguet avait bien raison de lancer en boutade que « le moyen infaillible de rajeunir une citation est de la faire exacte ${ }^{1}$ ». Voici deux exemples.

2 Avec la collaboration d'un doctorant russe, Dmitry Shatalov, j'ai démontré dans un article récent ${ }^{2}$ que l'auteur de la citation « Les traducteurs sont les chevaux de poste de la culture ${ }^{3}$ » est Alexandre Pouchkine et non Joseph de Maistre, comme l'ont cru à tort Pierre-François Caillé ${ }^{4}$ et plusieurs auteurs à sa suite. Nos recherches nous ont permis de rectifier la formulation française (Pouchkine parle de « chevaux de poste » et non de " chevaux de trait ») et de remonter à la source du fragment: le manuscrit d'Eugène Onéguine (1830).

Les pires ennemis de la citation, écrivions-nous, sont ceux qui, par paresse, ne vérifient pas leurs sources ou citent de deuxième, de troisième voire de quatrième main.

Une autre citation sur la traduction, d'Ernest Renan cette fois, a aussi été malmenée : sa formulation est erronée, sa source jamais citée. Edmond Cary la fait connaître en 1956 dans La traduction dans le monde moderne : « Une œuvre non traduite n'est qu'à demi publiée ${ }^{5} . »$ 


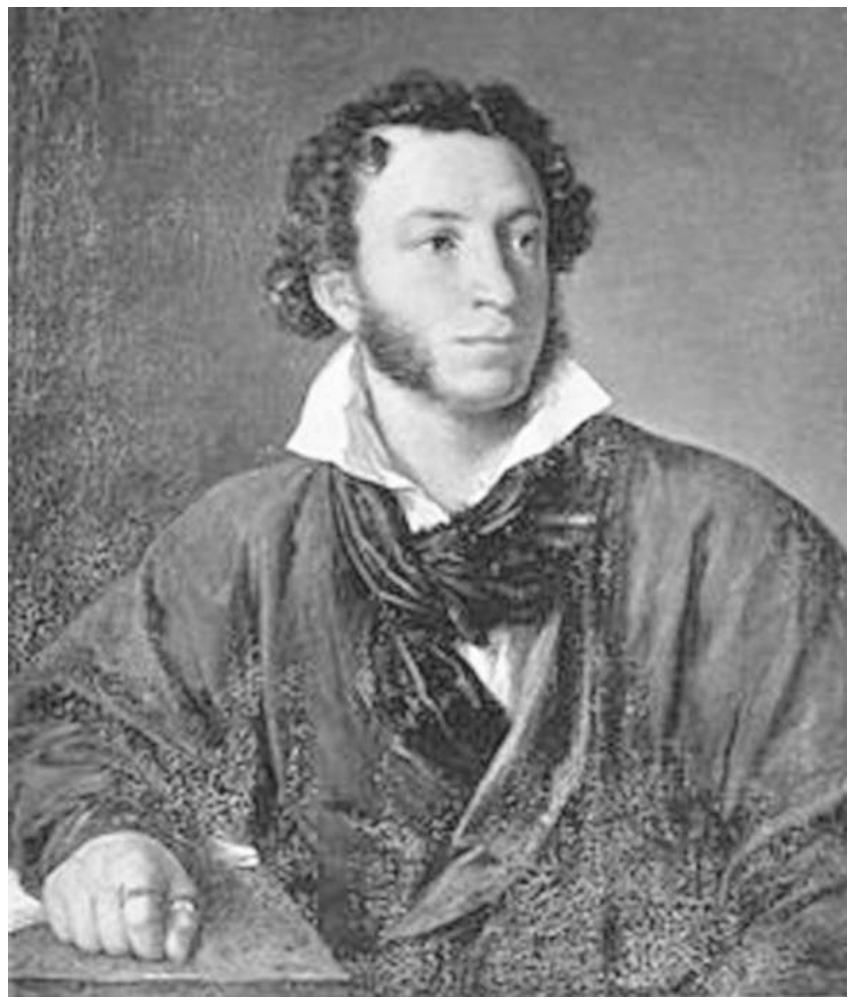

Alexandre Pouchkine

(portrait par Vassili Tropinine)

Parmi ceux qui l'ont utilisée, Jean-Claude Margot la cite d'après Cary en $1979^{6}$. Antoine Berman la reprend en 1984 dans L'épreuve de l'étranger, mais d'après Das Problem des Übersetzens ${ }^{7}$ publié sous la direction de Hans J. Störig. En la transcrivant, il introduit une légère variante par rapport à la version de Cary: «Une œuvre non traduite n'est publiée qu'à demi ${ }^{8}$ ». Aucun de ces auteurs ne renvoie à une publication de Renan. Sans la référence, comment vérifier laquelle des formulations est la bonne ? Comment avoir la certitude que la citation est bien de Renan?

5 La mention des sources fait souvent l'objet de l'indifférence ou de la négligence des auteurs. C'est pourtant dans le corps vivant du texte d'où elle est extraite qu'une citation est irriguée de tout son sens, et il est parfois important de s'y reporter. Un traducteur connait l'importance significative du contexte.

6 N'ayant pu retrouver la citation de Renan dans son œuvre considérable, malgré des recherches intensives et la consultation de spécialistes, j'avais dû me résigner à l'inclure "sous toutes réserves" dans mon dictionnaire La traduction en citations ${ }^{9}$, incapable de lui délivrer un certificat d'authenticité et de paternité. 


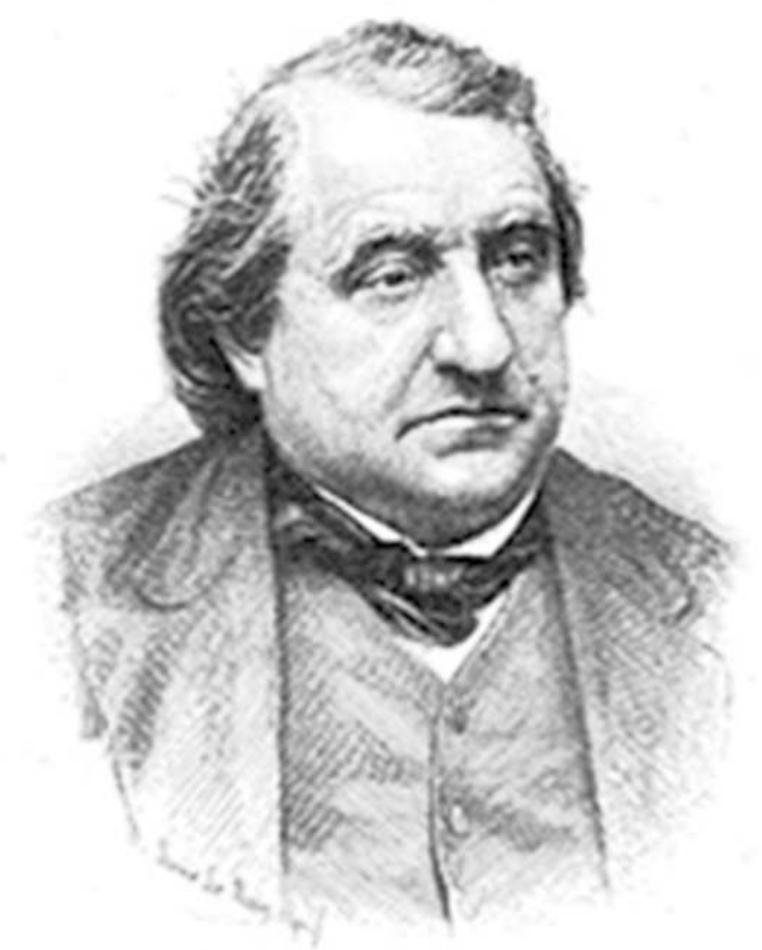

Ernest Renan

(gravure à l'eau forte de Louis Le Nain)

7 Or, le hasard a voulu que je tombe sur cette même citation en lisant l'Histoire des traductions en langue française, $\mathrm{XIX}^{\mathrm{e}}$ siècle ${ }^{10}$, ouvrage remarquable par son ampleur, la finesse de ses analyses et la qualité de ses synthèses. Ce vaste panorama original est publié sous la direction d'Yves Chevrel, Lieven D'hulst et Christine Lombez. Rompus aux exigences du travail scientifique rigoureux, ces chercheurs aguerris ont cité la source de l'extrait comme il se doit. Du coup, ils en ont rétabli la formulation exacte : « [1]'école française a raison de ne pas se départir de ce principe qu'un texte non traduit n'est qu'à demi publié11 ${ }^{11}$. Renan, qui affirme ici l'utilité et l'importance de la traduction, parle non pas d'une « œuvre », mais d'un " texte », mot dont le périmètre sémantique est plus large. Son apophtegme s'applique à tout genre de textes traduits et pas seulement aux œuvres littéraires.

me année, l'auteur de L'origine du langage reprend la même idée dans une autre recension : « un texte oriental n'est réellement publié que quand il est traduit ${ }^{12}$ ». Alliée naturelle de l'édition, la traduction parachève la publication de tout travail intellectuel. Idée lumineuse.

En comparant les traducteurs à des «chevaux de poste», Pouchkine disait sensiblement la même chose : chaque fois qu'un texte (littéraire ou non) est traduit dans une nouvelle langue, il entame une autre étape de son voyage universel, ce qui représente un progrès pour une culture, une civilisation.

Voilà donc deux citations qui valorisent le travail des traducteurs. Elles méritaient d'être « rajeunies » en étant bien référencées. 


\section{NOTES}

1. Rapportée par André Chaumeix dans son discours de réception à l'Académie française, le 30 avril 1931.

2. «Joseph de Maistre ou Alexandre Pouchkine? La confusion de Babel », L'Actualité langagière, vol. 9, $\mathrm{n}^{\circ}$ 4, 2013, p. 14-20. Aussi en ligne sur Termium Plus'

3. La langue russe autorise aussi la traduction « ... de la civilisation ».

4. « Avant-propos », Babel, vol. 1, n 1, 1955, p. 3.

5. Genève, Georg, p. 10.

6. Traduire sans trahir, Lausanne, Éditions L'Âge d'homme, p. 13.

7. Darmstadt, Wissenschaftliche Buchgesellschaft, 1963, p. vIII.

8. Paris, Gallimard, p. 283.

9. Ottawa, Les Presses de l'Université d'Ottawa, 2007, XxxIV-396 p.

10. Lagrasse, Verdier, 2012, p. 1247.

11. Ernest Renan, "L'Espagne musulmane », Journal des débats politiques et littéraires, 31 août 1853, p. 3. Aussi dans Euvres complètes, Paris, Calmann-Lévy, t. II, 1948, p. 522.

12. Ernest Renan, «Voyages d'Ibn-Batoutah», Journal des débats politiques et littéraires, 14 décembre 1853, p. 2. Aussi dans Euvres complètes, t. II, p. 531.

\section{AUTEUR}

\section{JEAN DELISLE}

Jean Delisle, traducteur et terminologue, titulaire d'un doctorat de la Sorbonne Nouvelle (Paris III) est professeur émérite à l'Université d'Ottawa où il a fait carrière de 1974 à 2007. Auteur et coauteur d'une vingtaine d'ouvrages, il a été traduit dans une quinzaine de langues. La pédagogie et l'histoire de la traduction sont ses deux champs de spécialisation. 